\title{
La mise en scène d'Astrée dans la Prolusio de Justitia gloriosè victrix, ac iniquè victa, Seu D. Stanislaus Episcopus Cracoviae de Carolus Kolczawa s.j. (1713)
}

Les allégories ${ }^{1}$ sont souvent mises en scène dans les drames de la Première Modernité. ${ }^{2}$ Les jésuites s'inscrivent dans cette tradition, notamment dans l'ouverture de leurs pièces ou dans les chœurs. Ces apparitions sont propices à des scènes chantées, voire dansées. Astrée est ainsi mise en scène à l'ouverture d'un drame en trois actes composé par Carolus Kolczawa au Clementinum de Prague et publié en 1713: Justitia gloriosè victrix, ac iniquè victa, Seu D. Stanislaus Episcopus Cracoviae. Cette pièce, consacrée à l'assassinat en 1079 de Stanislas de Szczepanóv, ${ }^{3}$ évêque de Cracovie, victime du roi de Pologne Boleslaus II, participe de l'entreprise de glorification des grands noms, notamment de l'Église catholique, victimes de la violence d'un souverain. Même si cette pièce a été écrite comme exercice rhétorique dans le cadre d'un établissement scolaire, elle a été conçue comme si elle pouvait être représentée. Nous tâcherons donc, dans cette contribution, de montrer combien la Prolusio de ce drame a été écrite selon des

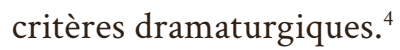

Comme le révèle la didascalie initiale, la scène d'ouverture est un affrontement (Astræa [...] ab Iniquitate oppugnatur), plus exactement une altercation entre deux allégories. Selon les mythes antiques, Astrée, une divinité fille de Zeus et de la

\footnotetext{
1 Nous remercions vivement Gabriella Körömi pour son invitation à participer au volume collectif publié en l'honneur de Márta Zsuzsanna Pintér. C’est un honneur pour nous de joindre cet article à tous les témoignages de très grande estime pour la richesse des recherches menées sur le théâtre par Márta Zsuzsanna Pintér et pour son dévouement dans l'organisation, au sein de son université, de nombreux colloques internationaux dont nous gardons tous un excellent souvenir.

2 Nous renvoyons à Jean-Pierre Bordier et André LASCOMBes (dir.), Dieu et les dieux dans le théâtre de la Renaissance, Actes du XLV Colloque International d'Études Humanistes, CESR Tours, 1-6 juillet 2002, (Turnhout: Brepols, 2006).

3 Voir Monika Siama, „Le palimpseste hagiographique de la Pologne du haut Moyen Âge: l'espace et le temps du culte de saint Stanislas de Szczepanovo", Revue des Études Slaves 79/1-2 (2008): 35-52.

4 On ne peut exclure que des scènes de la pièce, sinon la pièce entière, aient été jouées, même partiellement réécrites pour être adaptées à la scène, dans le cadre du Clementinum ou d'un autre collège jésuite de Bohême.
} 
Justice, a quitté la Terre, horrifiée par les forfaits commis par les hommes. ${ }^{5}$ Elle est devenue la constellation de la Vierge (vers 3: Decus, jubárque siderum). Iniquité, quant à elle, est une allégorie dont le nom, à lui seul, montre qu'elle est l'antithèse d'Astrée. Astrée, portant sans doute un vêtement caractéristique, commence donc par se présenter en citant son nom dès le premier vers pour que les lecteurs, ou les éventuels spectateurs, comprennent qui est le personnage seul sur scène au début du drame. Une machinerie permettrait sans doute de faire descendre sur scène le personnage pour signifier qu'Astrée quitte le Ciel pour revenir sur Terre le temps de la représentation (rursum coelo devolans). Ensuite, le monologue d'Astrée est interrompu, au vers 25, par l'arrivée d'Iniquité qui exprime sa surprise et son indignation de voir Astrée revenue sur Terre. Une altercation suit sous forme de stichomythies ou de couples de vers. Ainsi, après un ton solennel durant les vers 1 à 24 , se succèdent sarcasmes (vers 25 et 61-62 par exemple), menaces (vers 35-36 et 37-38 par exemple), arguments péremptoires (vers 41-42 par exemple), ordres (vers 48-49 par exemple), imprécations (vers 57 par exemple).

Iniquité joue le rôle d'un démon rappelant que le Ciel est le royaume de Dieu, mais que la Terre est l'espace des crimes, c'est-à-dire du péché. Alors qu'Astrée, au début de son monologue, fait trois fois référence à la Terre (vers 1, 7, 10), Iniquité se hâte de revendiquer la Terre pour elle (quatre références dans ses répliques: vers $25,32,34,42)$. À ses yeux, Dieu outrepasserait donc ses droits en intervenant sur Terre. Ainsi, quand Astrée proclame: Mundum gubernat providè,/ Unáque justè dirigit, Iniquité répond: Cælum gubernet providè,/ Nihílque terris imperet. La substitution de Caelum à Mundum permet à Iniquité de rectifier la profession de foi d'Astrée. De même, à la réplique suivante, à la suite de la déclaration d'Astrée (Stant cuncta jussu Numinis), Iniquité se contente de substituer deux mots pour limiter la puissance de Dieu (terra au lieu de cuncta, puis criminis au lieu de Numinis): Stat terra jussu criminis. Cet exercice d'école (la substitution de termes tout en gardant la structure métrique ainsi que les sonorités) révèle l'aisance oratoire d'Iniquité, qui sait répondre aux arguments de son adversaire.

La scène se joue, entre Ciel et Terre, dans un cimetière, plus précisément autour d'une tombe. Il s'agira de faire revenir à la vie un certain Petrus, dont les biens sont l'objet d'un différend judiciaire au cours duquel l'évêque et le roi s'opposent l'un à l'autre. Le procès ne pourrait être réglé que par une révélation de Petrus lui-même. La tombe est même la scène centrale des scènes 10 et 11 de l'acte I du drame : l'évêque fait ouvrir le tombeau et convoque le défunt au tribunal ; cette scène annonce aussi le martyre imminent de l'évêque, qui sera assassiné devant l'autel où il célèbre la messe (acte III, scène 22). Mais le crime sera châtié, puisque

\footnotetext{
5 Cf., par exemple, Virgile, Géorgiques, II, v. 473-474; Ovide, Métamorphoses, I, v. 149-150; Pseudo-
} SÉNĖQUE, Octavie, v. 423-425. 
l'on apprend, dès la Prolusio, qu'Astrée revient sur Terre pour punir les crimes des rois (vers 8 : Plexura Regum crimina). La scène liminaire du tombeau préfigure donc la dernière scène de la pièce. Par ailleurs, l'affrontement entre les deux allégories préfigure l'affrontement entre le roi et l'évêque.

Le roi Boleslaus incarne l'Iniquité puisqu'il veut la mort de Stanislaus par crainte de voir sa propre «iniquité» révélée. Par opposition, Stanislaus incarne la Justice, donc Astrée, dans l'intrigue. Ainsi, la Prolusio et les chœurs jouent sur le plan allégorique le combat entre le roi et l'évêque au long de l'intrigue: les chœurs à la fin des actes I et II mettent en scène un affrontement lyrique entre Iniquité et Astrée (fin de l'acte I), entre Inquité escortée de Dolus et Furor d'un côté et Astrée de l'autre (fin de l'acte II). Un chœur, peu avant la fin de l'acte III, met en scène Némésis, dont la venue était annoncée aux vers 58-59 de la Prolusio (Irata Nemesis vindice / Flagro sequetur crimina.). Le titre de la pièce exprime ce double plan : le personnage principal est à la fois Justitia et Stanislaus.

Le drame de Carolus Kolczawa développe amplement les sources historiques (ou plutôt hagiographiques) mentionnées dans l'Argumentum: le livre 8 de l'Historia Bohemica de Jan Dubravius. En comparant l'intrigue avec le récit des pages de Jan Dubravius, le lecteur entre dans le laboratoire d'écriture du dramaturge jésuite. Nous apprenons, au long des deux pages consacrées à la mort du roi Boleslaus II : 6

[p. 223.] Nam sub idem tempus hic ipse Deus justissimus, impietatem Boleslai Poloniæ Tyranni, qua se, per insectationem sui Pontificis S. Stanislai, dein per necem ejusdem parricidialem impiaverat justa evidentique vindicta ultus est, Regno illi per Rom. Pontificem adempto, actoque in exilium [p. 224] et in tantam amentiam dejecto, ut vagus sylvas pererraret: à suis denique canibus devoratus esse traditur. Sanctitatis porrò divi Stanislai, inauditum prius, tunc verò à cunctis, qui in aula regis aderant, oculis conspectum, hoc argumentum fuerit, quod Petrum quendam triennio exacto vita functum, sepultumque ad ferendum veritati testimonium contra Regem, jus Ecclesiæ oppugnantem, ad vitam revocaverit, deductum sua manu ante tribunal Regium constituerit, pavitante quidem ad vocem ejus effero Rege, non tamen vitia ingenii emendante: quanquam \& Vratislaus, \& uxor ipsius Suatava, quæ Boleslai soror erat, invitare illum ad meliorem frugem non destiterint.

Cette source ne fait que mentionner le retour à la vie d'un certain Petrus (preuve de la sainteté de l'évêque) pour que celui-ci apporte son témoignage contre le roi (constituant l'intrigue de l'acte I du drame); mais ces mêmes pages omettent les circonstances de l'assassinat de Stanislaus. Même si l'histoire était bien connue,

6 Nous avons consulté l'édition de 1687 (Francoforti: impensis J. G. Steck). 
surtout après la canonisation de Stanislaus en 1253, Carolus Kolczawa s'est appuyé sur d'autres sources, tout en laissant à son imagination la liberté de multiplier les rebondissements et les machinations au long des actes II et III.

On relève un souci permanent de la dramaturgie chez le professeur et poète dramatique. Un détail est révélateur: l'expression uale supremum (vers 45-47) signifie souvent, sur un ton pathétique, un dernier adieu entre deux êtres qui s'aiment. ${ }^{7}$ Cette expression, dénuée ici de tels affects, joue le rôle d'une didascalie interne: elle annonce la fin de la scène et la séparation des deux personnages. Mais, alors qu'Iniquité s'attend à ce qu'Astrée rejoigne le Ciel, celle-ci se réfugie dans un tombeau, vraisemblablement pour préfigurer la scène où Petrus, un noble polonais, reviendra parmi les vivants pour faire triompher la vérité et l'évêque. Cet effet de surprise, ou coup de théâtre, explique donc la présence d'un tombeau sur la scène (vers 54 et 56: in hoc sepulchro nobili, un vers répété pour marquer son importance) et sous-entend qu'Astrée, messagère de Dieu, sera présente, même invisible, lors de la scène où Petrus sera rappelé à la vie. La Prolusio permet de mettre en place un double espace qui caractérisera toute l'intrigue : espace terrestre et surnaturel (celui du Ciel et celui du monde des défunts). La Terre est l'espace théâtral de l'affrontement entre le Ciel et les Enfers.

Les allusions à la Gigantomachie complètent ce tableau d'une mise en scène surnaturelle. Si Astrée s'arroge le droit de descendre sur Terre, pour Iniquité les Géants sont en droit de remonter des Enfers. Tel est le sens de la double proclamation d'Iniquité (vers 37-38 et 60-63). Typhoée, quoique écrasé sous l'Etna, ${ }^{8}$ est le «modèle» même de l'hybris à l'encontre des dieux du panthéon antique. Cette pièce, qui met en scène un procès à l'acte $\mathrm{I}$, revendique donc la légitimité du pouvoir religieux (fas, au vers 17) et civil (æquum, au vers 17): l'ordre du droit (ordo iuris, au vers 18) peut ainsi être garanti. Cette scène délivre aussi un enseignement aux élèves du collège jésuite: Astrée demande de ne pas être ébloui par les généalogies et les trésors des puissants (vers 15-16); la justice se doit également de ne pas être partisane (vers 11-14).

Enfin le caractère spectaculaire de cette scène ne saurait être perçu sans l'explication d'un autre effet théâtral : la Prolusio relève du lyrisme puisqu'elle est composée non en trimètres iambiques, mais en dimètres iambiques acatalectiques. Le dimètre iambique acatalectique présente des iambes aux pieds pairs. Par exemple, le premier vers (Astræa terris exulans) présente le schéma métrique suivant: spondée premier, iambe deuxième, spondée troisième, iambe quatrième. Le dernier pied peut d'ailleurs, dans un dimètre iambique, être un pyrrhique (deux brèves). Les substitutions sont possibles aux pieds impairs. Ainsi le vers 35 (Tonantis ira

7 Ainsi lors de la séparation définitive entre Orphée et Eurydice. Cf. Ovide, Métamorphoses, X, v. 62. 8 Cf. Ovide, Fastes, I, v. 573 et IV, v. 491. 
fulmina) présente un iambe premier et un iambe troisième. Cette scène est donc vraisemblablement chantée et peut-être conçue avec des déplacements rythmés. Ce duel met ainsi en scène la confrontation entre l'harmonie, garantie par la Justice, et le risque du chaos, dessein d'Iniquité. Ce modèle métrique structure, par exemple, le deuxième chœur de Iephthes de George Buchanan (vers 341-360) et trois chœurs de Tobias de Petrus Vladeraccus (vers 765-796, 1169-1192, 1447-1472), des chœurs composés selon le modèle hymnique d'Ambroise, de Prudence ou de la liturgie catholique. ${ }^{9}$ Carolus Kolczawa s'éloigne cependant de cette inspiration pour dramatiser, à l'aide de ce même dimètre iambique acatalectique, l'apparition d'Astrée en composant non une hymne chrétienne, mais une altercation dans le but de célébrer la justice divine. Il renouvelle ainsi le genre de l'hymne en dramatisant celui-ci. Il se situe aussi dans l'héritage des Psaumes puisque le terme iniquitas a été vraisemblablement choisi par Carolus Kolczawa parce que celui-ci apparaît très fréquemment dans ce livre, dans 56 psaumes. ${ }^{10}$ Le combat oratoire entre Astrée et Iniquité se situe donc dans le prolongement du lyrisme des psaumes.

Le drame s'achève sur l'assassinat de l'évêque par le roi lui-même et ses conseillers. Le roi ordonne que le corps de Stanislaus soit démembré et offert aux rapaces. La dernière scène voit, comme la tradition le rapporte, un aigle, lui aussi descendu du ciel, protéger le corps de Stanislaus. Un ballet, permettant la participation d'un plus grand nombre d'élèves au spectacle, conclut la pièce ; leur danse imite, d'une certaine façon, le vol des aigles : mira Aquilarum chorea. L'adjectif mira qualifie à la fois l'effet produit sur les éventuels spectateurs, mais aussi l'atmosphère générale de l'intrigue, du merveilleux à la terreur et inversement. À l'apparition surnaturelle d'Astrée lors de la Prolusio fait donc écho ce ballet d'aigles à la fin de la dernière scène : deux moyens scéniques pour mettre en scène l'intervention de la Providence dans la vie des hommes. À Astrée mise à mort par Iniquité succède l'aigle comme médiateur de Dieu. L'effet théâtral, saisissant, prouve que Carolus Kolczawa composait certes ses pièces comme exercices rhétoriques, mais qu'il les concevait en même temps comme pouvant être représentées.

Exercitationes dramaticae Caroli Kolczawa Societatis Jesu, Pars IV, Pragae, Typis Univers. Carolo-Ferdinandeæ in Collegio Soc. JESU ad S. Clementem Anno 1713.

9 Voir Jean-Louis Charlet, Métrique latine humaniste. Des préhumanistes padouans et de Pétrarque au XVI siècle, Travaux d'Humanisme et Renaissance (Genève: Droz, 2020), 370-375.

10 Voir les psaumes $5,6,7,8,10,15,16,17,25,26,27,30,31,35,36,37,38,39,40,44,48,50,51$, $52,54,56,57,58,61,63,65,68,71,72,73,74,78,81,84,88,89,91,93,100,102,105,106,108$, $118,124,128,129,138,139,140,142$. 


\title{
[p. 275] JUSTITIA
}

gloriosè victrix, ac iniquè victa,

$\mathrm{Seu}$

D. STANISLAUS

Episcopus Cracoviæ.

\begin{abstract}
ARGUMENTUM.
D. Stanislaus Episcopus Cracoviensis in æquissimæ suæ causæ defenssionem ad vivos reuocavit ${ }^{11}$ Petrum nobilem Polonum, eúmque stitit tribunali regio, sententiam pro Episcopo dicenti. Hunc veridicum testem cùm in malo obstinatus Rex Boleslaus refellere non valeret, ferrô refutavit, Sanctúmque Præsulem ad aras immaniter trucidavit. Dubravius li. 8.
\end{abstract}

\section{[p. 276] INTERLOCUTORES.}

Stanislaus Episcopus.

Boleslaus Rex.

Petrus defunctus.

Potocus Regni Primas.

Jablonus et Sapinus Regni Optimates.

Zolenus et Holenus Regni Senatores.

Vasinus et Mosinus Petri hæredes.

Vespillo

CHORUS.

Astræa.

Nemesis.

Iniquitas.

Furor.

Dolus.

11 Nous substituons revocavit à la leçon éditée recovavit en nous appuyant sur l'extrait précédemment cité du livre 8 de l'Historia Bohemica de Jan Dubravius: [...] quod Petrum quendam triennio exacto vita functum, sepultumque ad ferendum veritati testimonium contra Regem, jus Ecclesiæ oppugnantem, ad vitam revocaverit, deductum sua manu ante tribunal Regium constituerit. Par ailleurs, le participe présent oppugnantem deviendra oppugnatur dans le résumé initial de la Prolusio. 


\section{[p. 277] PROLUSIO.}

Astræa rursum coelo devolans, ab Iniquitate oppugnatur. Quare in monumento defunctorum se condit, plaudente in terris iniquitate.

Astr. Astræa terris exulans,

Colo recepta, vixeram

Decus, jubárque siderum,

Ignara noxæ fœderum.

Jam pulchra cœli culmina

Mutare jussa, lurida

Reviso terrae limina,

Plexura Regum crimina;

Hæc est voluntas Numinis,

Terrae regentis machinam.

10

Non ulla sceptri gratia,

Sed nec odiorum jurgia,

In vota debent impia

Juris bilancem flectere.

Sum cæca, nunquam stemmata,

Nullasque gazas intuens.

Quod fas, \& æquum judicat;

Id ordo juris approbat.

Sint virtuosis præmia:

Poenas reportent crimina.

Urnam movebit Æacus,

Utì Themistis ${ }^{12}$ imperat.

Pœnâ innocentes eximet;

Culpæ reos coarguet,

[p. 278] Iniq. Rursúmne terras visere

Themis fugata deligit?

12 La leçon Themistis est une variante de Themis, comme l'a indiqué Magdaléna Jacková en note du vers 325 de la pièce intitulée Telo furoris amoris potior vis. Voir Magdaléna JaCKоVÁ, Nejmírnějsi Pallas Hry určene gramatikálním tř́dám jezuitských gymnáziú, Theatrum Neolatinum, Latinske divadlo v ceskych zemich, svazek II (Praha: Academia, 2016), 302. 
Astr. Sic Numen altum præcipit.

Iniq. A lege nostra deficit.

Astr. Mundum gubernat providè, Unáque justè dirigit.

Iniq. Cælum gubernet providè,

Nihílque terris imperet.

Astr. Stant cuncta jussu Numinis.

Iniq. Stat terra jussu criminis.

Astr. Tonantis ira fulmina

In vasta mittet crimina.

Iniq. Surgent gigantum robora, Trahéntque cœlo Numina.

Astr. Nihil potestas terrea

In alta tentet sidera.

Iniq. Sit ergo cœlum Numini.

Sit terra solùm crimini.

Aut vise rursus aethera:

Vel eris furori victima.

Astr. Iniquitati dicere

Vale supremum præcipit

Mundi universi conditor.

Iniq. Illum revise, \& cælicas

Duntaxat oras incole.

Astr. Sub mortuorum lugubri

Secura degam marmore.

Iniq. In mortuorum sedibus

Astræa degat mortua. 
[p. 279] Astr. In hoc sepulchro nobili Noctes quietas exigam.

Iniq. In hoc sepulchro nobili

Noctes agas ignobiles.

Astr. Irata Nemesis vindice

Flagro sequetur crimina.

Iniq. Terrore lude parvulos,

Non strenuos Typhoëos.

Iniquitatis gloria

Ridet Tonantis prælia.

Argument:

Stanislas, évêque de Cracovie, rappela à la vie, pour défendre sa cause très légitime, Pierre, un noble polonais, et il le fit comparaître devant le tribunal royal : sa déclaration donnait raison à l'évêque. Mais, comme, s'obstinant dans le mal, il ne pouvait pas réfuter ce témoin de la vérité, le roi Boleslaus répondit par le fer et il assassina avec barbarie le saint prélat devant les autels. Lire le livre 8 de Dubravius.

Prélude ${ }^{13}$

Astrée, volant pour redescendre du Ciel, est assaillie par Iniquité. C'est pourquoi elle se cache dans un tombeau, tandis que, sur la Terre, l'iniquité applaudit.

Astrée

Moi, Astrée, exilée de la Terre, reçue au Ciel, je vivais jusqu'à présent

gloire et éclat des astres, sans connaître le tort causé aux lois.

Aujourd'hui j'ai reçu l'ordre de quitter

les beaux sommets du Ciel pour revenir voir

les seuils livides de la Terre,

afin de châtier les crimes des rois;

13 Le terme «prolusio» signifiait notamment, dans la Rome antique, «préparation à un combat». Le terme est ici doublement justifié: ouverture lyrique de la pièce et préfiguration du combat entre le roi et l'évêque. 
telle est la volonté de la Puissance divine, qui dirige la structure de la Terre.

Aucune faveur venant du sceptre, mais même aucune querelle due à des haines ne doivent faire incliner la balance du droit vers des vœux impies.

Je suis alors privée de la vue, car je ne scrute jamais généalogies ou trésors.

La justice divine et humaine, voilà ce que l'ordre du droit approuve.

Aux vertueux les récompenses!

Que les crimes rapportent leurs châtiments!

Éaque agitera son urne, selon la volonté de Thémis. ${ }^{14}$

Elle préservera les innocents du châtiment; elle prouvera la culpabilité des accusés...

\section{Iniquité}

Thémis, pourtant mise en fuite, a-t-elle choisi

de revenir rendre visite à la Terre?

Astrée

Oui, selon la prescription de la haute Puissance divine.

Iniquité

Elle contrevient à la règle que nous avons fixée.

Astrée

Elle gouverne le monde avec providence

et en même temps elle le dirige avec justice.

Iniquité

Qu'elle gouverne le Ciel selon sa providence

sans donner aucun commandement à la Terre!

14 On trouve déjà sous la plume de Carolus Kolczawa, dans le premier volume des drames publié à Prague en 1703, une réflexion sur Thémis dans un dialogue entre deux personnages (Carolus KolcZAwa, Ambitio regnandi seu Corbin, Acte I, scène XIII, 63). 
Astrée

Tout tient ferme sur ordre de la Puissance divine.

Iniquité

La Terre tient ferme sur ordre du crime.

Astrée

Le courroux du Tonnant lancera

ses foudres contre les crimes monstrueux.

\section{Iniquité}

L'élite des Géants se dressera

et délogera du Ciel les Puissances d'en haut.

\section{Astrée}

Que le pouvoir terrestre ne lance

aucune attaque contre les astres élevés!

\section{Iniquité}

À la Puissance divine donc le Ciel!

Au crime et à lui seul la Terre !

Regagne l'éther;

sinon à la fureur tu seras offerte comme victime. ${ }^{15}$

\section{Astrée}

Le Créateur de l'univers tout entier

prescrit de dire un adieu définitif

à Iniquité.

\section{Iniquité}

$\mathrm{Va}$ retrouver celui-ci et contente-toi

d'habiter les rivages des cieux.

\section{Astrée}

Sous le marbre lugubre des défunts, puissè-je vivre ${ }^{16}$ en sécurité!

15 Cette menace sera mise à exécution quand Furor et Dolus s'associent à Iniquitas pour combattre Astrée dans le chœur qui clôt l'acte II.

16 Il peut s'agir d'un subjonctif présent, mais aussi d'un futur. 
Iniquité

Dans les demeures des défunts

qu'Astrée vive morte!

Astrée

Dans ce noble tombeau,

puissè-je passer des nuits en paix!

Iniquité

Dans ce noble tombeau,

puisses-tu passer des nuits infâmes!

Astrée

Le courroux de Némésis, de son fouet vengeur, poursuivra les crimes.

Iniquité

De Typhoées tout-petits, sans énergie,

60

fais d'eux le jouet de la terreur.

La gloire d’Iniquité, quant à elle, se rit des combats du Tonnant.

\begin{abstract}
Carolus Kolczawa s.j. published in 1713 at the Clementinum in Prague a drama about the death of St Stanislas bishop of Cracow in 1079. In the prelude of the drama, Astræa and Iniquitas are arguing about who is the most powerful on Earth. In this paper we give the Latin text of this Prelude with a French translation. But first we comment on the scene which was conceived as a spectacular dispute.
\end{abstract}

